\title{
Dietary Intake of Creatine in Children Aged 0-24 Months
}

\author{
Darinka Korovljev Nikola Todorovic Valdemar Stajer Sergej M. Ostojic \\ Applied Bioenergetics Laboratory, Faculty of Sport and PE, University of Novi Sad, Novi Sad, Serbia
}

\section{Dear Editor,}

Creatine is often emphasized as an important component of early life nutrition. Derived from diet or synthesized endogenously, creatine contributes to normal child health and development by supporting cellular energy homeostasis [1]. Being a component of human breast milk, creatine could also be obtained by infant formulas and animal-based foods in early childhood [2]. However, daily creatine intake in very young children currently remains unknown. Here, we quantified the amount of creatine consumed through a regular diet among US children aged $0-2$ years using data from the 2017 to 2018 National Health and Nutrition Examination Survey (NHANES).

Dietary intake information from NHANES 2017-2018 Dietary Data component was extracted for children aged 0-24 months (597 participants, 320 boys and 277 girls; age $10.4 \pm 7.1$ months). To calculate creatine intake, we first identified creatine-containing foods (e.g., milk and milk products; meat, poultry, fish, and mixtures) using 8-digit food codes from the US Department of Agriculture (USDA) proxy-interview entries for individual foods. We subsequently recorded the gram weight of each food component containing creatine (US Department of Agriculture codes from 11100000 to 28522000 ) and calculated the net intake of those foods for each child by merging all relevant food items on a daily basis. Individual values for

karger@karger.com

(c) 2021 S. Karger AG, Basel

www.karger.com/anm

Karger" total grams of creatine consumed per day were computed using the average amount of creatine $(0.20 \mathrm{~g} / \mathrm{kg}$ for milkbased foods and $3.88 \mathrm{~g} / \mathrm{kg}$ for meat-based sources) across all creatine-containing food sources. The daily creatine intake excluded the amount of creatine taken through breast milk since no data about the volume consumed was obtainable from the NHANES database.

Nonhuman milk, infant formulas, and other milk products were a source of creatine in 438 out of 597 children (73.4\%), and creatine-containing meat-based foods were consumed by 205 children (34.3\%). A total of 149 children (24.9\%) were exclusively fed with breast milk (number of breastfeeding sessions 1-31 per day). The mean dietary intake of creatine across the sample was 0.28 $\pm 0.24 \mathrm{~g} /$ day ( $95 \%$ confidence interval, from 0.25 to 0.30 ). The daily creatine intake categorized into quartiles ranges from 0.00 to $0.12 \mathrm{~g}$ ( 1 st quartile), $0.13-0.20 \mathrm{~g}$ (2nd quartile), $0.21-0.34 \mathrm{~g}$ (3rd quartile), and 0.35-1.46 g (4th quartile) (Fig. 1).

Our results showed a high variability of daily creatine intake among US children aged $0-2$ years, with nonhuman milk identified as the primary food source of creatine. The average creatine intake of $0.28 \mathrm{~g}$ per day found in this population appears to be approximately $1 / 4$ of dietary creatine requirements for adults $(1 \mathrm{~g} /$ day $)$ [3]. This implies that the dietary intake of creatine in very young
Correspondence to:

Sergej M. Ostojic, sergej.ostojic@ chess.edu.rs 


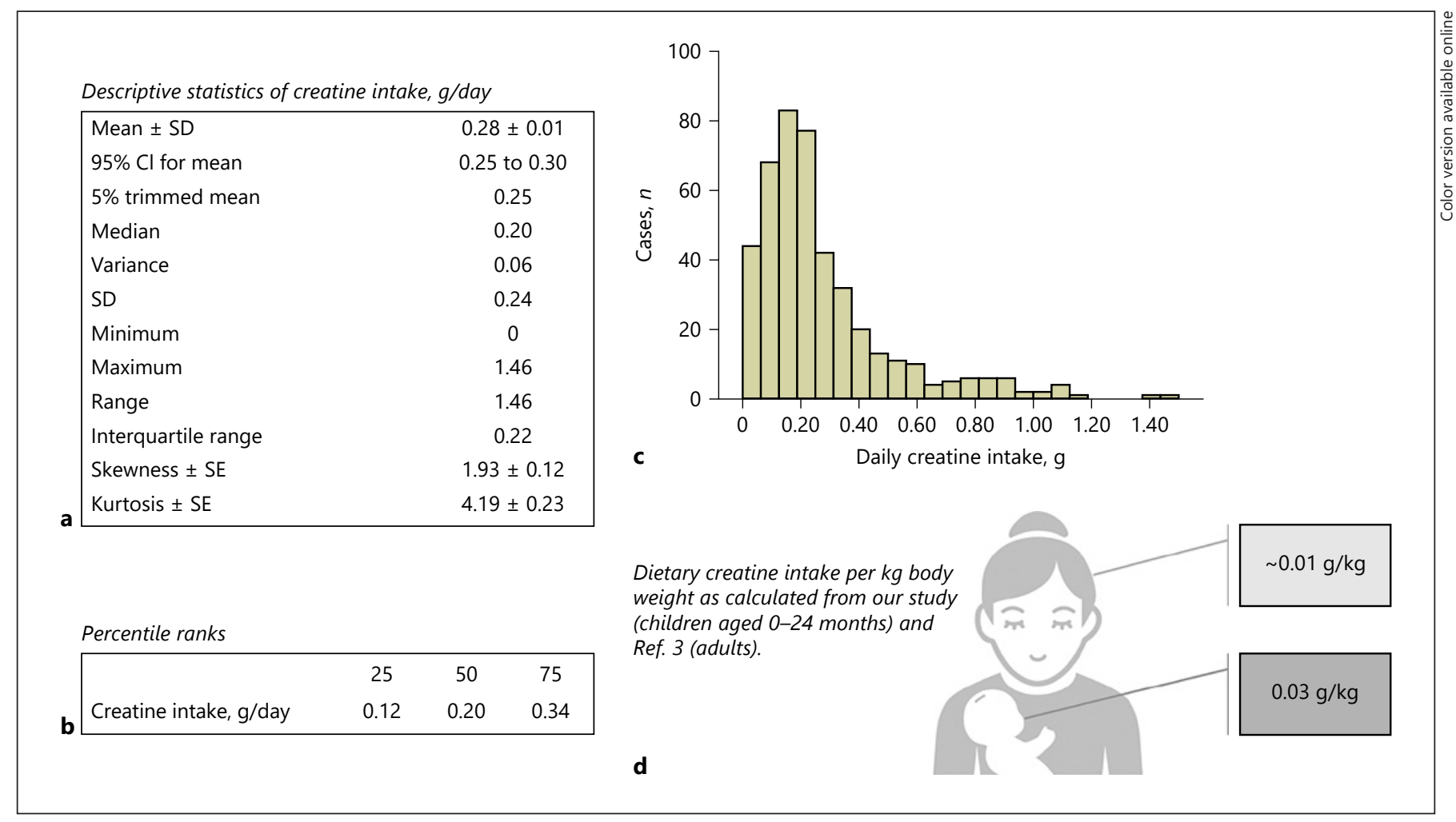

Fig. 1. Descriptive statistics (a), percentile ranks (b), histogram (c), and calculated relative consumption of children versus adults (d) for daily dietary intake of creatine across NHANES 2017-2018 children aged 0-24 months $(n=597)$. SE, standard error; CI, confidence interval; SD, standard deviation.

children is roughly 3 times larger than that of the adult population in terms of body size (Fig. 1). A sufficient intake of creatine via food may play a critical role in optimal brain development due to possible limitations of endogenous creatine synthesis machinery at a young age $[3,4]$. Further, studies are thus highly warranted to evaluate the link between dietary creatine intake and neurodevelopment in early childhood.

\section{Conflict of Interest Statement}

D.K., N.T., and V.S. declare no conflict of interest. S.M.O. serves as a member of the Scientific Advisory Board on creatine in health and medicine (AlzChem LLC). S.M.O. owns patent "Sports Supplements Based on Liquid Creatine" at European Patent Office (WO2019150323 A1), and active patent application "Synergistic Creatine" at UK Intellectual Property Office (GB2012773.4). S.M.O. has served as a speaker at Abbott Nutrition, a consultant of Allied Beverages Adriatic and IMLEK, and an advisory board member for the University of Novi Sad School of Medicine, and has received research funding related to creatine from the Serbian Ministry of Education, Science, and Technological Development, Provincial Secretariat for Higher Education and Scientific Re- search, AlzChem GmbH, KW Pfannenschmidt GmbH, ThermoLife International LLC, and Monster Energy. S.M.O. is an employee of the University of Novi Sad and does not own stocks and shares in any organization. The funders had no role in the design of the study; in the collection, analyses, or interpretation of data; in the writing of the manuscript; or in the decision to publish the results.

\section{Funding Sources}

No funding has been received.

\section{Author Contributions}

D.K.: conducted research; analyzed data; wrote paper draft; revised the paper. N.T.: conducted research; analyzed data; wrote paper draft; revised the paper. V.S.: conducted research; analyzed data and performed statistical analysis; revised the paper. S.M.O.: designed research (project conception, development of overall research plan, and study oversight); analyzed data and performed statistical analysis; wrote paper draft; and had primary responsibility for final content. All the authors read and approved the final manuscript. 


\section{References}

1 Muccini AM, Tran NT, de Guingand DL, Philip M, Della Gatta PA, Galinsky R, et al. Creatine metabolism in female reproduction, pregnancy and newborn health. Nutrients. 2021 Feb 2;13(2):490.

2 Edison EE, Brosnan ME, Aziz K, Brosnan JT. Creatine and guanidinoacetate content of human milk and infant formulas: implications for creatine deficiency syndromes and amino acid metabolism. Br J Nutr. 2013 Sep 28; 110(6):1075-8.
3 Brosnan JT, Brosnan ME. Creatine: endogenous metabolite, dietary, and therapeutic supplement. Annu Rev Nutr. 2007;27:24161.

4 Dickinson $\mathrm{H}$, Ireland $\mathrm{ZJ}$, Larosa $\mathrm{DA}$, O'Connell BA, Ellery S, Snow R, et al. Maternal dietary creatine supplementation does not alter the capacity for creatine synthesis in the newborn spiny mouse. Reprod Sci. 2013 Sep; 20(9):1096-102. 